RESEARCH ARTICLE

\title{
Indices for measuring performance of smallholder rubber units
}

\author{
Wasana Wijesuriya ${ }^{1 *}$, R.O. Thattil ${ }^{2}$ and S. Samita ${ }^{3}$ \\ Rubber Research Institute of Sri Lanka, Dartonfield, Agalawatte. \\ 2 Post Graduate Institute of Agriculture, University of Peradeniya, Peradeniya. \\ 3 Department of Crop Science, Faculty of Agriculture, University of Peradeniya, Peradeniya.
}

Revised: 05 October 2006; Accepted: 14 February 2007

\begin{abstract}
Performance indices are in existence for annual crops, but no such attempt has been reported for rubber. This study developed performance indices for rubber, to assess growth, yield and field conditions using data collected from smallholder surveys in the three districts, Kegalle, Kalutara and Ratnapura. These indices were Growth Performance Index (GPI), Yield Performance Index (YPI) and Field Assessment Score (FAS). GPI had a right skewed distribution and fitted best to the Beta type-2 distribution. GPI had a mean of 0.99 and a variance of 0.09 . YPI for Kegalle, Kalutara and Ratnapura districts varied from 0.16 to 3.83 (mean $=0.99$, variance $=0.36), 0.22$ to $4.35($ mean $=1.06$, variance $=0.44)$ and 0.23 to 3.61 (mean $=1.09$, variance $=0.33)$, respectively. Log normal distribution was found to be the best fit for YPI, irrespective of location. FSA distributed according to a normal distribution with a mean of 57.3 and a variance of 186 . These performance indices provide a basis to compare rubber lands and provide a basis to identify potential farmers to introduce new technologies. These indices together with their distributional properties can be effectively used for analyses of risks associated with introducing new technologies for different groups of farmers and economic efficiency of rubber lands.
\end{abstract}

Key words: Distributional properties, field assessment score, growth performance index, rubber, yield performance index.

\section{INTRODUCTION}

The two measurements that assess the performance of rubber during immature and mature stages are growth and yield of the crop, respectively. Growth of rubber is measured by the tree circumference (commonly known as girth) at $1.2 \mathrm{~m}$ from the highest point of the bud union. Growth during the immature stage ensures early attainment of tapping ( $50 \mathrm{~cm}$ girth at $1.2 \mathrm{~m}$ height) and high yields during the mature stage.

Latex yield of rubber is the measure that indicates the performance of a rubber land during mature stage. Yield of rubber is generally measured as $\mathrm{g} /$ tree/tapping or $\mathrm{kg} / \mathrm{ha} /$ year. Rubber yield depends on various environmental and management factors, age and different rubber clones.

Developing performance indices for growth and yield become important as it provides a basis for comparing rubber lands on their performance, which are of different ages. Subsequently, these indices can be effectively used for risk analysis and economic efficiency analysis. One of the important aspects of risk assessment is to fit appropriate probability distribution functions to longterm yield data, representing system response to variability in environmental factors and management practices ${ }^{1}$. In many risk analyses, the normal distribution has been applied to determine probabilities of unfavourable events. However, in many cases, alternative distribution functions would better fit the yield data ${ }^{1}$.

Studying the distribution of Growth Performance Index (GPI) is important for rubber as there may be differences in the distributions with the management practices. It can also be regarded as a risk analysis subjected to different objectives viz. eligibility for subsidy payment and possibility of reducing immature period.

Yield performance indices of any crop can be used to identify the potential farmers to introduce new technologies.In annual crops like paddy, yield performance indices are calculated by dividing the observed yield by the average yield of all farmers in a certain village under the study. The methodology on developing yield performance indices and applications on paddy are well established and documented ${ }^{2-4}$. This kind of index is not applicable to perennial crops like rubber, where the sample of fields may comprise of different ages of crop and when the yield also varies with the age of the

\footnotetext{
* Corresponding author
} 
crop. Hence, there is a basic need to develop indicators to assess performance of the rubber holdings, which may be useful in many ways in monitoring and planning

This paper introduces performance indices, a novel approach for rubber, based on a study carried out with the objectives to develop (a) a GPI taking into consideration the growth requirement for the eligibility for subsidy payments/early attainment of growth (b) a Yield Performance Index (YPI) for rubber based on the average yield profile of smallholdings and (c) a Field Assessment Score (FAS) based on field evaluation on major agronomic recommendations in the immature stage. The distributional properties of these developed indices were studied and an attempt was also made to relate the field conditions with the growth performance of the holdings.

\section{METHODOLOGY}

\section{Collection of data}

Girth data: Girth data were collected in a survey during the latter part of the year 2001 (Survey 1) covering all the Rubber Development Officer (RDO) ranges in the Kalutara district which included 160 immature holdings. The sampling technique used in this survey was a stratified random system with 4 strata with equal allocation. The RDO divisions were separated into 4 strata based on the performance of the holdings determined using the subsidy payment information ${ }^{5}$.

Yield data: This study employed the data collected under the project "Interactions between the Environment, Society and Technology - (INTEREST)" carried out by the Rubber Research Institute of Sri Lanka (RRISL) during the period 2002 to 2004 (Survey 2) in Kegalle, Kalutara and Ratnapura districts ${ }^{6}$. The yield data used in this study were from the rapid sample survey, which covered nine selected villages with 653 smallholder farmers in the above districts. The yield was collected as number of sheets produced per day with the average weight of rubber sheets to estimate the rubber yield when yield records were not available with the farmers.

Condition of smallholder fields: The data collected in technical evaluation of 160 immature holdings in Survey 1 using a field evaluation sheet (Table 1) were also employed in this study.

\section{Construction of indices}

Growth Performance Index (GPI): For the smallholder (owns extents less than 4 ha), maintaining a healthy uniform stand is of importance due to two reasons. One is that, to qualify for a regular subsidy payment, there should be a continuous improvement in growth. The other objective is the reduction of immature period to bring the holding into tapping in 5 years ${ }^{7}$, which is clearly a possible task with adoption of recommended practices. The proposed index is as follows.

$G P I_{i k}=G T O B_{i k} / G T E X_{i k}$

Where, $G T O B_{i k}=$ observed girth, $G_{T E X}=$ expected girth and $G P I_{i k}=$ growth performance index at age $i$ of the $\mathrm{k}^{\text {th }}$ holding. Expected values change with the objective; i.e. (A) subsidy requirement or (B) reduced immature period as given in Table 2 . The index based on girth requirement for subsidy payments was used for distribution fitting in this study.

Yield Performance Index (YPI)

$\mathrm{YPI}_{\mathrm{ij}}=\left(\mathrm{Yd} \mathrm{Oob}_{\mathrm{ij}} / \mathrm{Yd} \mathrm{d}_{-} \mathrm{v}_{\mathrm{j}}\right)$

Where: YPI $_{\mathrm{ij}}$ is the yield performance index of $\mathrm{i}^{\text {th }}$ holding at $j^{\text {th }}$ age, Yd_ob ${ }_{i j}=$ observed yield of the $i^{\text {th }}$ holding at $j^{\text {th }}$ age, and $Y d_{-}{ }^{a} v_{j}=$ average yield for $j^{\text {th }}$ age.

The fitted yield distribution with age of the smallholdings in the 3 districts $^{5}$ was employed to estimate $\mathrm{Yd}_{-} \mathrm{av}_{\mathrm{j}}$.

Field Assessment Score (FAS): The FAS was calculated for the fields based on an evaluation on technical recommendations of the immature rubber holdings falling into the 4 main field operations viz., (i) land preparation (ii) field establishment (iii) ground cover management and (iv) other maintenance activities. A score is assigned ${ }^{5}$ to each field according to a field evaluation sheet as given in Table 1.

\section{Distribution fitting}

The theoretical distributions fitted to the constructed indices were: a) Normal, b) Log normal, c) Gamma, and d) Beta type 2 .

\section{a) Normal distribution}

The density function for the Normal distribution ${ }^{8}$ is:

$f(x)=\frac{1}{\sigma \sqrt{2 \pi}} e^{-1 / 2\left(\frac{x-\mu}{\sigma}\right)^{2}}$.

The parameters to be estimated are $\mu$ and $\alpha$ The sample skewness is 0 with variance $6 / \mathrm{n}$ and the sample kurtosis is 0 with sampling variance $24 / \mathrm{n}$. 
Table 1: The field sconng sheet with allotted marks for each freld actrusty

\begin{tabular}{|c|c|c|c|c|}
\hline & \multicolumn{2}{|c|}{ Field operation } & \multicolumn{2}{|c|}{ Max score } \\
\hline \multicolumn{3}{|c|}{1 Land Preparation } & & 30 \\
\hline \multirow[t]{4}{*}{ A } & & Crop sanitation & 15 & \\
\hline & I & Fence & & \\
\hline & II & Unnecessary trees (Inside holding/periphery) & & \\
\hline & III & Removal of roots & & \\
\hline \multirow[t]{5}{*}{ B } & & Soll conservation (drain/terrace/platform) & 15 & \\
\hline & I & Number & & \\
\hline & II & Dimensions & & \\
\hline & III & Whether on contours & & \\
\hline & IV & Maintenance & & \\
\hline \multicolumn{3}{|c|}{2 Freld Establishment } & & 25 \\
\hline A & \multicolumn{2}{|c|}{ Clone (budded/recommended/non mixed) } & 5 & \\
\hline B & \multicolumn{2}{|c|}{ Recommended spacing } & 5 & \\
\hline $\mathrm{C}$ & \multicolumn{2}{|c|}{ Recommended stand } & 10 & \\
\hline D & \multicolumn{2}{|c|}{ Planting method (contour \& depth) } & 5 & \\
\hline \multicolumn{3}{|c|}{3 Ground Cover Management } & & 20 \\
\hline A & \multicolumn{2}{|c|}{ Weeding ( $3^{\prime}$ diameter around the plant) } & 5 & \\
\hline \multirow[t]{5}{*}{ B } & B 1 & Cover crop & 15 & \\
\hline & & Steps taken to establısh & & \\
\hline & & Type (legume/mixed legume/other) & & \\
\hline & & Percentage distribution & & \\
\hline & & Growth & & \\
\hline \multirow[t]{4}{*}{ B } & B-2 & Intercropped lands & 15 & \\
\hline & & Recommended crop & & \\
\hline & & Recommended spacing & & \\
\hline & & Maintenance & & \\
\hline B & $\mathrm{B}-3$ & Cover crop + Intercrop & 15 & \\
\hline \multicolumn{3}{|c|}{4 Other Maıntenance Actıvitıes } & & 25 \\
\hline \multirow[t]{4}{*}{ A } & & Plant growth & 15 & \\
\hline & & Girth accordıng to age, clone and climate & & \\
\hline & & Uniformity & & \\
\hline & & Free of vacant patches & & \\
\hline \multirow[t]{4}{*}{ B } & & Control of Branching & 5 & \\
\hline & & Removal of stock shoots & & \\
\hline & & Removal of lateral shoots & & \\
\hline & & Branchıng above $8^{\prime}$ & & \\
\hline $\mathrm{C}$ & & Disease control & 5 & \\
\hline \multicolumn{3}{|c|}{ TOTAL SCORE } & & 100 \\
\hline
\end{tabular}

Source Wijesuriya, B W 5 
Table 2: Required girth of rubber for (a) eligibility for regular subsidy payments and (b) early attainment of tapping in 5 years

\begin{tabular}{|c|c|c|}
\hline \multirow[t]{2}{*}{ Year } & \multicolumn{2}{|c|}{ Required girth $(\mathrm{cm})$ under different objectives } \\
\hline & $\begin{array}{l}\text { Eligibility for regular subsidy } \\
\text { payments }\end{array}$ & $\begin{array}{c}\text { Early attainment of tappable } \\
\text { girth in } 5 \text { years }\end{array}$ \\
\hline 1 & 6 & 10 \\
\hline 2 & 10 & 20 \\
\hline 3 & 16 & 30 \\
\hline 4 & 25 & 40 \\
\hline 5 & 32 & $50^{*}$ \\
\hline 6 & $50^{*}$ & \\
\hline
\end{tabular}

* $60 \%$ of the trees in a holding should obtain a girth of $50 \mathrm{~cm}$ to start tappping

\section{b) Log normal distribution}

The $\log$ normal distribution assumes that $\log (\mathrm{X})$ is normally distributed with mean $\mu$ and variance $\sigma^{2}$. An additional location parameter $a$ can be included in the model and the normal distribution is fitted to $\log (\mathrm{X}-a)$. The density function ${ }^{8}$ is:

$f(x)=\frac{1}{x \sqrt{2 \pi \sigma}} \exp \left[-\left(\log _{e} x-\mu\right)^{2} / 2 \sigma^{2}\right] I_{(0, \infty)}(x)$

Where, $-\alpha<a<b<\alpha, \beta>0$

mean $=\exp \left[\mu+\frac{1}{2} \sigma^{2}\right] \quad$ and

variance $=\exp \left(2 \mu+\sigma^{2}\right)\left\{\exp \left(\sigma^{2}\right)-1\right\}$.

c) Gamma distribution

The density function for the 2-parameter model is:

$f(x)=b^{k} x-1 \frac{e^{-b x}}{\Gamma(k)}$

where, $\quad \Gamma(k)=\int_{0}^{\infty} x^{k-1} e^{-x} d x$

with mean $\mu=k / b$ and variance $k b^{-2}$. The special cases are when $k=1$, gamma distribution becomes an exponential distribution, if $b=1 / 2$ it is a $\chi^{2}$ distribution with $2 k$ degrees of freedom and if $b=1$, gamma tends to be the standard normal as $k$ reaches infinity.

d) Beta type 2 distribution

The beta distribution is suitable for fitting proportions or ratios. Two types are available, where type 1 is a two- parameter model restricted to values in the range $0<x<1$. The beta type 2 is suitable for any positive continuous data and has 3 parameters. The density function ${ }^{9}$ for this type is:

$$
f(x)=\frac{b^{p_{x} p-1}}{(1+b x)^{p+q} \mathbf{B}(p, q)} \times>0 .
$$

The distribution has mean $\mu=p /\{b(q-1)\}$ and variance $\mathrm{V}=\mathrm{p}(\mathrm{p}+\mathrm{q}-1) /\left\{\mathrm{b}^{2}(\mathrm{q}-1)^{2}(\mathrm{q}-2)\right\}$

The DISTRIBUTION directive in Genstat 7 was employed in distribution fitting. The goodness of fit of the fitted distributions was assessed by the associated probability values of residual deviances, which have an asymptotic chi-squared distribution with the specified degrees of freedom?.

\section{RESULTS AND DISCUSSION}

\section{Descriptive statistics and distributional properties of GPI}

GPI has a positively skewed distribution as shown in Figure 1. GPI values varied from 0.39 to 2.13 with a mean of 0.99 and the coefficient of variation (CV) is 30 . Table 3 gives the deviances of the fitted distributions and the estimates of theoretical distributions. The results presented in Table 3 also suggest that GPI departed from normality, as the deviance observed was 35.19 on 23 d.f. resulting an approximate $\chi^{2}$ probability of $<0.05$. Other 3 models satisfactorily fit the data, according to the probability values of resulting deviances shown in Table 3 . Beta type 2, Gamima and Log normal distributions fit to the GPI values satisfactorily as the deviances are not significant. Among them, the model Beta type 2 had the highest p-value and hence was the best fit. 


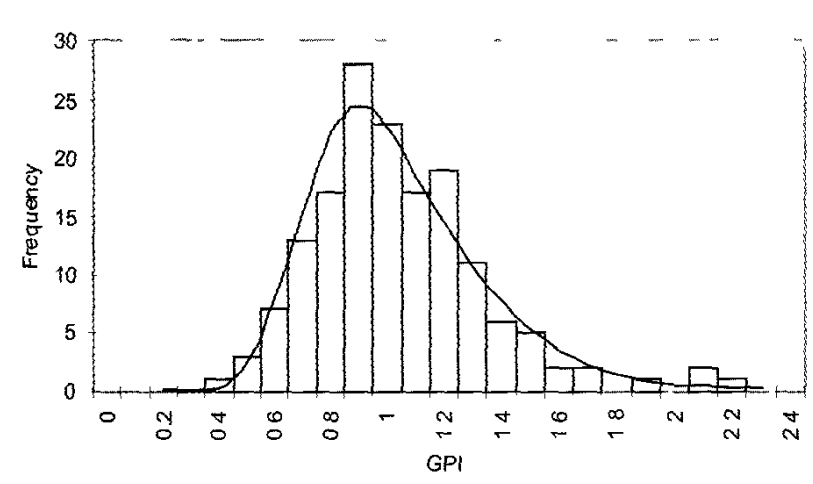

Figure 1: Distribution of the GPI

Table 3: The results of fittung different distributions to Growth Performance Index (GPI)

\begin{tabular}{|c|c|c|}
\hline Distribution & Estimates of parameters & $\begin{array}{c}\text { Deviance and approx. } \\
\chi^{2} \text { probability }\end{array}$ \\
\hline Normal & $\begin{array}{ll}\mu=0.99 & (0.02) \\
\sigma=0.30 & (0.02)\end{array}$ & $\begin{array}{r}3519 \text { on } 23 \text { d.f. } \\
\text { p }<0.05\end{array}$ \\
\hline Log Normal & $\begin{array}{ll}\mu=-0.06 & (0.02) \\
\sigma=0.31 & (0.02)\end{array}$ & $\begin{array}{r}20.98 \text { on } 23 \mathrm{df} \\
\mathrm{p}=0.60\end{array}$ \\
\hline Gamma & $\begin{array}{ll}k=10.69 & (1.18) \\
b=10.85 & (1.22)\end{array}$ & $\begin{array}{r}19.21 \text { on } 23 \mathrm{~d} f \\
p=0.70\end{array}$ \\
\hline Beta type 2 & $\begin{array}{l}p=16.90(7.44) \\
q=33.33(13.48) \\
b=0.53(0.19)\end{array}$ & $\begin{array}{r}16.30 \text { on } 22 \text { d.f. } \\
p=0.80\end{array}$ \\
\hline
\end{tabular}

Note: Values in parentheses are corresponding standard errors.

\section{Descriptive statistics and distributional properties of YPI}

The mean of YPI in the Ratnapura district was 1.09 and followed by the Kalutara and Kegalle districts with 1.06 and 0.99 , respectively. The Ratnapura district had the lowest CV (52) while the districts Kegalle and Kalutara had CV values of 61 and 62 , respectively. In all 3 districts, YPI had positively skewed distributions. Table 4 presents the deviances and associated probabilities of the fitted distributions and the estimates of theoretical distributions. The probability levels $(p<0.001)$ observed in the 3 districts for the normal distribution suggest that YPI departs significantly from the normality. $\log$ normal distribution was found to be the best fit for all the districts and for pooled YPI according to the associated probability levels of the deviances presented in Table 4. The fitted Log normal distributions to YPI of the 3 districts are given in Figure 2.

\section{Descriptive statistics and distributional properties of FAS}

FAS has a mean of 57.3 and varied in the range 26 to 89 . The deviances and associated probabilities of the fitted distributions and the estimates of theoretical distributions are given in Table 5. The Normal distribution was found to be the best fit with a deviance of 16.6 on 17 d.f. $(p=0.5)$. The observed and fitted frequencies are depicted in Figure 3.

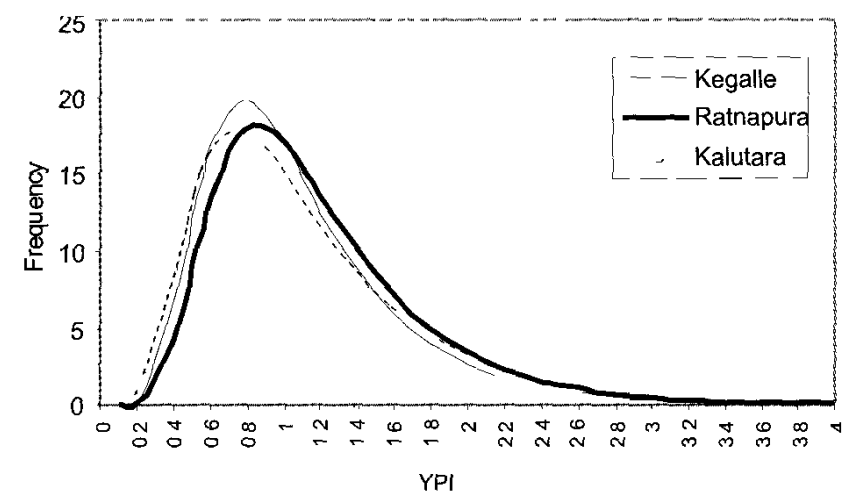

Figure 2: Fitted Log normal distributions to yield performance index (YPI) in 3 districts

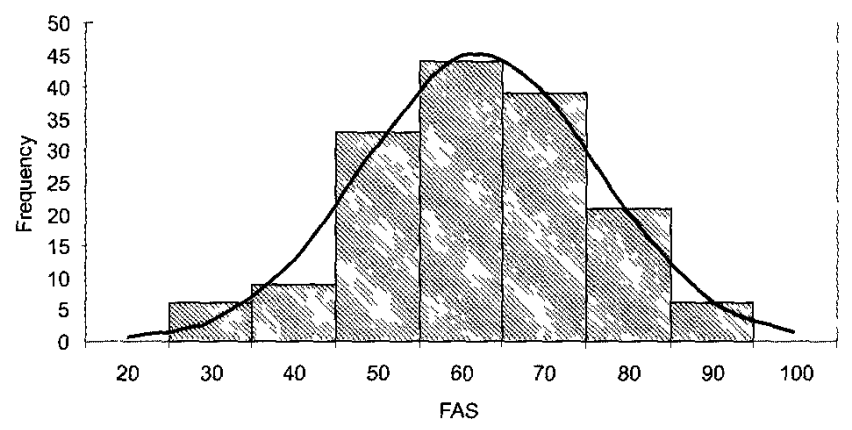

Figuire 3: Observed frequencies and fitted normal distribution to Field Assessment Score (FAS)

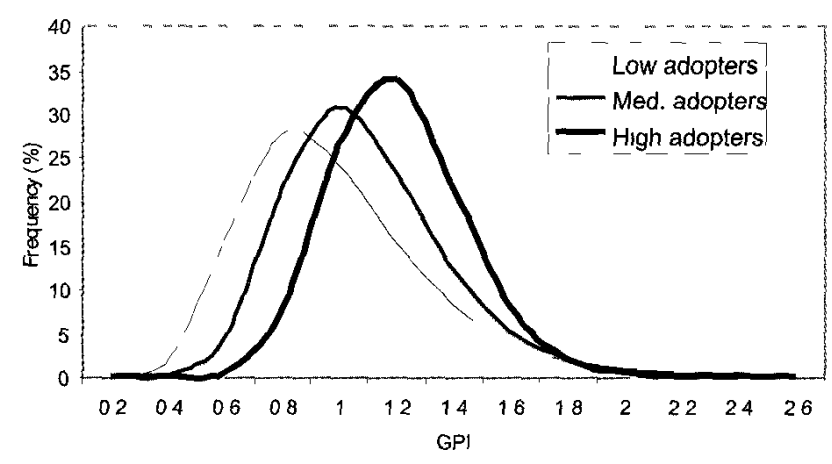

Figure 4: Fitted distributions of growth performance indices (GPI) for field assessment score (FAS) categories (low adopters and medium adopters - Log_normal, high adopters - normal) 
Table 4: The results of fitting different distributions to Yield Performance Index (YPI)

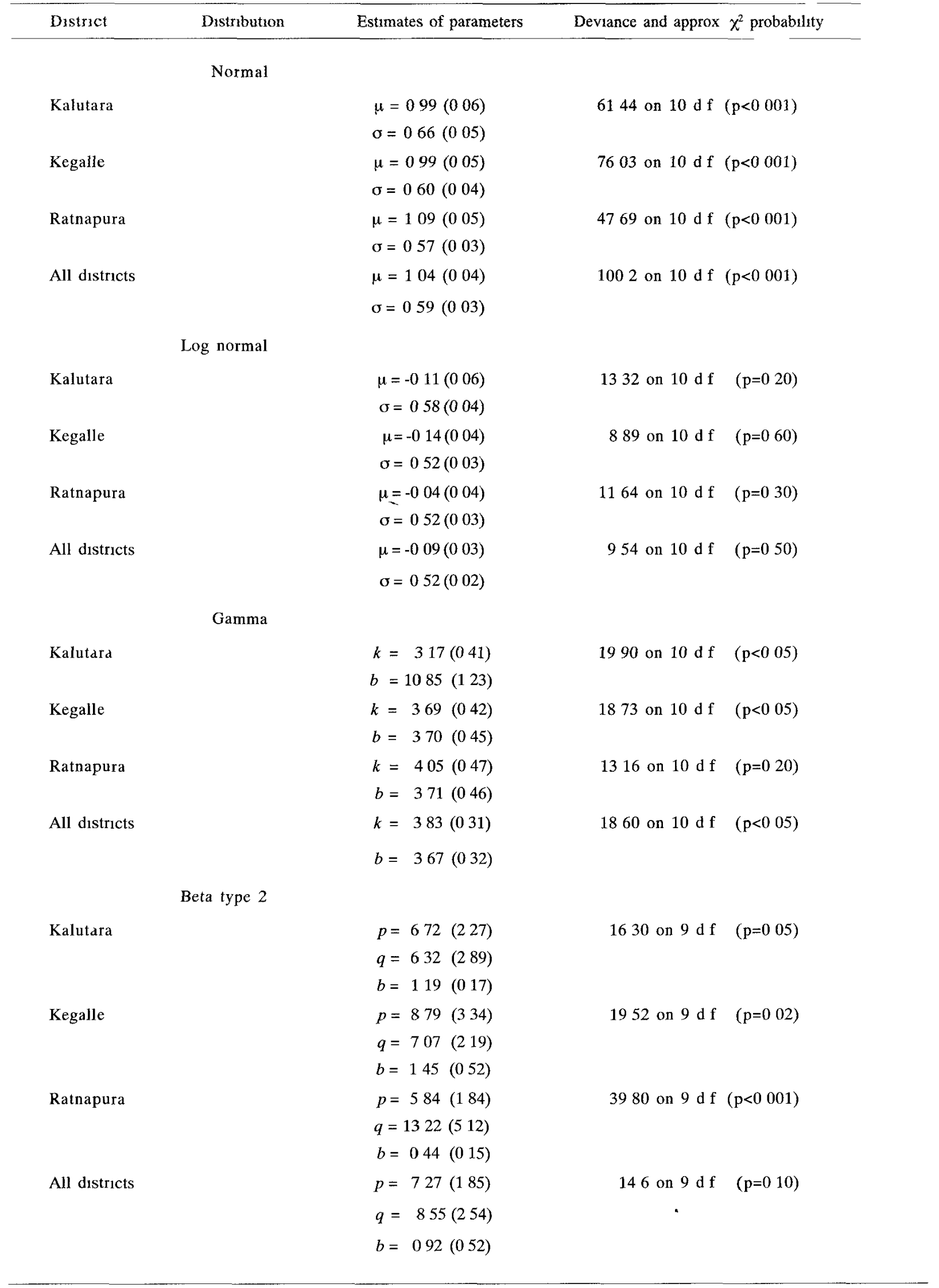

Note Values in parentheses are corresponding standard errors 


\section{Relationship between FAS and GPI}

FAS takes into account, all aspects of a rubber holding as indicated in Table 1, while growth performance indices only take into account the growth performance of the rubber holding. A positive relationship $(\mathrm{r}=0.48, \mathrm{p}<0.001$, $\mathrm{n}=160$ ) was found between FAS and GPI values derived from the rubber holdings in Survey 1 . The mean shifted towards right side with the increase of FAS. The coefficient of variation was highest in 'low adopters' and lowest in 'high adopters', which gives an idea of the uniformity of GPI in the group of 'high adopters'.

The Log normal distribution was the best fit to the GPI of both 'low adopters' and 'medium adopters' (Tables $6 a$ and $6 b$ ) while the GPI of category 'high adopters' fitted to normal distribution (Table $6 \mathrm{c}$ ). The fitted distributions of GPI for the 3 FAS categories are depicted in Figure 4.

Table 5: The results of fittıng different distributions to Field Assessment Score (FAS)

\begin{tabular}{|c|c|c|}
\hline Distribution & Estimates of pardmeters & Deviance and approx $\chi^{2}$ probability \\
\hline Normal & $\begin{array}{l}\mu=5731\left(\begin{array}{ll}1 & 08\end{array}\right) \\
\sigma=1359\left(\begin{array}{ll}0 & 76\end{array}\right)\end{array}$ & 166 on $17 \mathrm{df} p=050$ \\
\hline Log Normal & $\begin{array}{l}\mu=402\left(\begin{array}{ll}0 & 02\end{array}\right) \\
\sigma=026\left(\begin{array}{ll}0 & 01\end{array}\right)\end{array}$ & 3185 on $17 \mathrm{df} p<00$ \\
\hline Gammd & $\begin{array}{l}k=1632\left(\begin{array}{ll}1 & 82\end{array}\right) \\
b=028\left(\begin{array}{ll}0 & 03\end{array}\right)\end{array}$ & 2527 on $17 \mathrm{~d} f \mathrm{p}<010$ \\
\hline Beta type 2 & 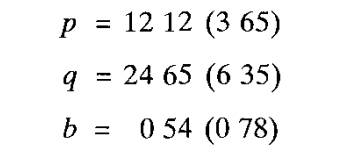 & 2451 on $16 \mathrm{~d} f \mathrm{p}<010$ \\
\hline
\end{tabular}

Note Values in parentheses are corresponding standard errors

Table 6a The results of fitting different distributions to GPI when FAS $<50$

\begin{tabular}{|c|c|c|}
\hline Distribution & Estımates of parameters & Deviance and approx $\chi^{2}$ probability \\
\hline Normal & $\begin{array}{l}\mu=089(006) \\
\sigma=038(004)\end{array}$ & 3086 on $17 \mathrm{~d}$ f $\mathrm{p}<002$ \\
\hline Log Normal & $\begin{array}{l}\mu=-018\left(\begin{array}{ll}0 & 05\end{array}\right) \\
\sigma=035\left(\begin{array}{ll}0 & 04\end{array}\right)\end{array}$ & 1394 on $17 \mathrm{df} p=070$ \\
\hline Gamma & $\begin{array}{l}k=741(153) \\
b=829(177)\end{array}$ & 1835 on $17 \mathrm{~d} \mathrm{f} \mathrm{p}=040$ \\
\hline Beta type 2 & 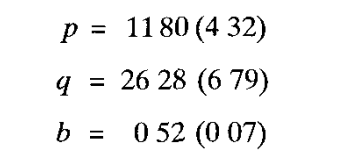 & 1398 on $16 \mathrm{df} p=060$ \\
\hline
\end{tabular}

Note Values in parentheses are corresponding standard errors 
Table 6b: The results of fitting different distributions to GPI when $50<$ FAS $<69$

\begin{tabular}{|c|c|c|}
\hline Distribution & Estimates of parameters & Deviance and approx. $\chi^{2}$ probability \\
\hline \multirow[t]{2}{*}{ Normal } & $\mu=0.99(0.03)$ & 21.75 on 17 d.f. $p=0.20^{\circ}$ \\
\hline & $\sigma=0.27(0.02)$ & $\cdot$ \\
\hline \multirow{2}{*}{ Log normal } & $\mu=-0.05(0.03)$ & 16.21 on 17 d.f. $p=0.50$ \\
\hline & $G=0.27(0.02)$ & \\
\hline \multirow[t]{5}{*}{ Gamma } & $k=13.86(2.19)$ & 18.47 on 17 d.f. $p=0.30$ \\
\hline & $b=13.97(2.25)$ & \\
\hline & $p=14.75(4.67)$ & 16.56 on 16 d.f. $p=0.40$ \\
\hline & $q=29.98(8.56)$ & \\
\hline & $b=0.52(0.15)$ & \\
\hline
\end{tabular}

Note: Values in parentheses are corresponding standard errors.

Table 6c: The results of fitting different distributions to GPI when FAS is $>=70$

\begin{tabular}{|c|c|c|}
\hline Distribution & Estimates of parameters & Deviance and approx. $\chi^{2}$ probability \\
\hline \multirow[t]{2}{*}{ Normal } & $\mu=1.11(0.04)$ & 6.39 on 17 d.f. $p>0.90$ \\
\hline & $\sigma=0.22(0.03)$ & \\
\hline \multirow[t]{2}{*}{ Log normal } & $\mu=0.08(0.04)$ & 8.59 on 17 d.f. $p>0.90$ \\
\hline & $\sigma=0.21(0.03)$ & \\
\hline \multirow[t]{2}{*}{ Gamma } & $k=23.29(5.53)$ & 9.65 on 17 d.f. $p>0.90$ \\
\hline & $b=1.09(5.06)$ & \\
\hline \multirow[t]{3}{*}{ Beta type 2} & $p=16.59(5.63)$ & 8.97 on 16 d.f. $p>0.90$ \\
\hline & $q=28.97(7.65)$ & \\
\hline & $b=0.59(0.15)$ & \\
\hline
\end{tabular}

Note: Values in parentheses are corresponding standard errors.

\section{CONCLUSION}

The index, GPI departed from normality and has a right skewed distribution and fitted best to the Beta type 2 distribution. GPI varied from 0.39 to 2.13 with a mean of 0.99. normal distribution was found to be the best fit for YPI. YPI varied from 0.16 to 3.83 (mean=0.99), 0.22 to 4.35 (mean=1.06) and 0.23 to 3.61 (mean=1.09) for the districts Kegalle, Kalutara and Ratnapura, respectively. FAS distributed according to a normal distribution with a mean of 57.3 and ranged from 26 to 89 . FAS had a significant positive relationship with GPI $(r=0.48, p<0.001)$. The proposed index, FAS is of practical use to extension workers to monitor the status of rubber lands and can be employed to evaluate the lands for the purpose of releasing subsidy payments.

Although the distribution of GPI is right skewed it was observed that when the distributions of GPI were studied under 'low', 'medium' and 'high' adopter categories, only 'low' and 'medium' categories departed from normality. The shifting of distributions of GPI towards right, when adoption levels change from 'low' to 'high' 
was observed for FAS. The GPI values under 'medium' and 'low' adopter categories can be fitted to Log normal distribution. The indices developed in this study are of practical value to analyze smallholder surveys. Further, the fitted distributions can be employed in economic studies such as assessing the risk and uncertainty and efficiency in the smallholder sector of rubber.

\section{References}

1 Rötter R., van Keulin H. \& Jansen M.J.W. (1997). Variations in yield response to fertilizer application in the tropics: I. Quantifying risks and opportunities for smallholders based on crop growth simulation. Agricultural Systems 53: 41-68.

2 Wijeratne M. \& Abeydeera I.R.N. (1995). Farmer performance in a rice-based farming system: differences between new and old systems. International Rice Research Notes 20(4): 21.

3 Wijeratne M. \& de Silva W.N. (2003). Farmer categorization based on farmer performance index: identifying the targets of agricultural extension. International Rice Research Notes 28(1): 72.

4 Pathirana U.P.N.S. \& Wijeratne M. (2003). Yield performance under the rice yaya demonstration program in Sri Lanka: a case study. International Rice Research Notes 28(2): 55 .

5 Wijesuriya B.W. (2004). Development of performance indicators and statistical techniques for analyzing participatory research in smallholder rubber units. $P h D$ thesis, Postgraduate Institute of Agriculture, University of Peradeniya, Peradeniya.

6 RRISL (2003). Review of the Biometry section, Annual Review, the Rubber Research Institute of Sri Lanka, Dartonfield, Agalawatta.

7 Seneviratne P. (2005). Production of planting material for rubber replanting and new planting programmes in Sri Lanka. Bulletin of the Rubber Research Institute of Sri Lanka 46: 17-24.

8 Mood A.M., Graybill F.A. \& Boes D.C. (1988). Introduction to the theory of statistics. McGraw-Hill Inc.

9 Genstat. (2003). The guide to Genstat 2. Statistics, Genstat for Windows, Seventh Edition, VSN International Ltd., Oxford, UK. 